Enfoques. Revista de Investigación en Ciencias de la Administración http://doi.org/10.33996/revistaenfoques.v3i9.54 No. 9 | Volumen 3 | Enero - Marzo 2019 http://revistaenfoques.org ISSN: $2016-8219$ pp. $66-82$

\section{MODELO PARA LA GESTIÓN DE RIESGOS EN PROYECTOS EJECUTADOS EN SITIOS REMOTOS POR CONTRATISTAS PETROLERAS DEL ESTADO ZULIA}

\author{
MODEL FOR RISK MANAGEMENT PROJECTS EXECUTED IN \\ REMOTE SITES BY OILCONTRACTORS OF ZULIA STATE
}

Jesús Villasmil

Artículo recibido octubre 2018 | Arbitrado noviembre 2018 | Publicado 01 de enero 2019

\section{Resumen}

El presente estudio tuvo como propósito plantear un modelo para la gestión de riesgos en proyectos ejecutados en sitios remotos por empresas contratistas del sector petrolero del estado Zulia, a fin de reducir el impacto de los riesgos. La investigación fue de tipo proyectiva, con un diseño de campo, transeccional, no experimental. Para el análisis de la información obtenida se emplearon como técnicas de tratamiento de datos la estadística descriptiva, específicamente frecuencia absoluta y porcentual y la medida de tendencia central media, los cuales indican que la población encuestada aborda medianamente el tema de riesgos en los proyectos, puesto que en muchos casos no cuentan con herramientas técnicas efectivas que permitan el levantamiento de información oportuna para tomar las acciones. Para finalizar se obtuvo que la variable gestión de riesgos puede verse afectada por las debilidades encontradas en el análisis de la dimensión situación de los riesgos, considerada como vital en el éxito o fracaso de un proyecto.

Palabras clave: Gestión de riesgos; proyecto; respuesta al riesgo; control de riesgos; sitios remotos

\begin{abstract}
The purpose of this study was to propose a model for risk management in projects executed in remote sites by contractors of the oil sector in Zulia state, in order to reduce the impact of risks. The research was projective, with a field design, transectional, not experimental. For the analysis of the information obtained, descriptive statistics, specifically absolute and percentage frequency and the measure of central tendency were used as data processing techniques, which indicate that the population surveyed fairly addresses the issue of project risks, since that in many cases they do not have effective technical tools that allow the collection of timely information to take the actions. Finally, it was obtained that the risk management variable can be affected by the weaknesses found in the analysis of the risk situation dimension, considered as vital in the success or failure of a project.
\end{abstract}

Key words: risk management; project; risk response; risk control; remote sites
Jesús Villasmil

jvillasmils@gmail.com

EHCOPEK-S.A., Universidad del Zulia, Venezuela

Ingeniero Mecánico, Universidad del Zulia, Venezuela, Magister en Gerencia de Proyectos industriales. Profesor universitario y experto en planificación de proyectos, auditor líder en sistemas de gestión de la calidad bajo la norma ISO 9000 . 


\section{INTRODUCCIÓN}

La dinámica empresarial de hoy en día ha cambiado por muchas razones, quizás la más importante ha sido mantenerse competitivos en el mercado, por ello, ya no es suficiente con cumplir con calidad los objetivos de un proyecto, sino que es necesario sobrepasar las exigencias del cliente consiguiendo resultados excepcionales. Las empresas actuales se ven obligadas a incursionar en nuevos paradigmas que las encaminen a buscar la mejora continua de sus procesos, minimizar los costos y optimizar los tiempos de ejecución de los proyectos.

De hecho, la gestión de los proyectos está condicionada a las características que estos poseen, puesto que nunca las condiciones temporales, operativas $y$ financieras son las mismas debido a factores externos e internos propios de la naturaleza de la obra a ejecutar. Aunado a esto, surgen los riesgos inherentes producto de la incertidumbre generada por la presencia de eventos que pudieran ser positivos o negativos y que por tal razón es necesario idear mecanismos de respuesta que permitan minimizar el impacto negativo o maximizar la influencia positiva sobre el desarrollo de las actividades del proyecto.

En efecto, en cada acción de la cotidianidad existe siempre un cierto grado de peligro $u$ oportunidad. De manera tal, en el mundo de la gerencia, toda operación o plan trae consigo inseguridad; en tal sentido, en los proyectos está implícita la probabilidad de que ocurran imprevistos durante su desarrollo. De esta manera, una alternativa para culminar de forma satisfactoria una obra o proyecto es la aplicación de la gestión del riesgo de forma estable, sólida y con una perspectiva lo suficientemente amplia para lograr el éxito de los elementos que conforman su ejecución.
Los proyectos son concebidos por el PMBOK (2004), como un esfuerzo temporal que se lleva a cabo para crear un producto, servicio o resultado único que tienen una naturaleza temporal, y tienen como finalidad alcanzar unos objetivos o metas, para lo cual se hace necesaria la planificación, control y monitoreo de todas sus actividades, sin embargo por su amplitud están expuestos a factores internos o externos que comprometen su éxito.

Asimismo, Cartay (1996) define al riesgo como una situación que puede tener influencias sobre los objetivos de un proyecto en las áreas de alcance, duración, costo y calidad. Para el mismo autor, la gerencia tiene la responsabilidad de hacer juicios formales así como tomar decisiones oportunas y apropiadas para conducir los proyectos hacia un destino exitoso. Tales medidas pueden ser tomadas dentro de condiciones de certidumbre total, si se cuenta con toda la información para pronosticar resultados altamente confiables.

De igual manera, Lledó y Rivarola (2007) explican que la gestión de los riesgos es el proceso sistemático de planificar, identificar, analizar, responder y controlar los riesgos del proyecto. Este proceso trata de maximizar la probabilidad de ocurrencia de los sucesos positivos y minimizar la probabilidad de ocurrencia de los sucesos adversos.

Lo anteriormente expuesto por los autores indica que la gestión de riesgo es un aspecto de suma importancia en la gerencia de proyecto, sin importar su tipo o característica. Es así como a finales de la década de los noventa varios países mostraron su interés en crear y participar en diferentes comisiones para abordar dicha temática, proponiendo nuevos modelos de control interno, que garanticen procesos más seguros y donde tanto el hombre como 
las organizaciones puedan desarrollarse armonicamente. (Coopers y Lybrand, 1997).

Con el correr de los años, diferentes instituciones relacionadas con el manejo del riesgo enfatizan la importancia de desarrollar esquemas de control que garanticen la buena marcha de las organizaciones y el resguardo de sus activos. Estas previsiones han sido las principales impulsoras de la gestión de riesgos que hoy forma parte de la cultura organizacional que existe en el mundo y muy particularmente en América Latina, como es el caso de Venezuela, donde a partir de 1999 mediante la promulgación de la nueva Constitución de la República Bolivariana de Venezuela, se da un cambio sustancial en cuanto al reconocimiento de derechos laborales y de protección atribuidos a la población trabajadora del país.

De esta manera, una alternativa para culminar de forma satisfactoria una obra o proyecto es la aplicación de la gestión del riesgo de forma estable, sólida y con una perspectiva lo suficientemente amplia para lograr el éxito de los elementos que conforman su ejecución.

En el caso específico de esta investigación, en las empresas contratistas del sector petrolero se desarrollan proyectos en zonas remotas caracterizadas por condiciones peculiares que aumentan la probabilidad de ocurrencia de situaciones no deseadas, por cuanto la Corporación Petróleos de Venezuela Sociedad Anónima (PDVSA), ha fijado una política de expansión y producción de hidrocarburos mediante acuerdos internacionales suscritos por el gobierno venezolano con países vecinos.

Esta situación enmarca el desarrollo de numerosos proyectos de la industria petrolera y del gas en sitios de difícil acceso (selvas, montañas o el mar) denominados sitios remotos. Por esta razón, especialmente en el estado Zulia, el $80 \%$ de las obras de infraestructura desarrolladas por Petróleos de Venezuela S.A., conjuntamente con empresas contratistas petroleras, ha presentado cambios de alcance, que ocasionan un impacto en el costo de las obras, tiempo de ejecución y diseño de las mismas.

Se reconoce como proyectos remotos aquellos que son ejecutados fuera de las instalaciones de la organización que los desarrolla, generalmente en zonas de difícil acceso y condiciones ambientales adversas que dificultan su normal desarrollo debido a la carencia de servicios públicos, proveedores, mano de obra calificada, entre otros.

Una de las problemáticas presentadas en el desarrollo de ingenierías tipo procura y construcción (PC), cuyos alcances están asociados a trabajos de rehabilitación, adecuación y mantenimiento de instalaciones ya existentes en estos sitios remotos, es que presentan un alto retraso evidenciado en proyectos sin culminar en diferentes etapas, planes sin respuesta a un gran portafolio de solicitudes para desarrollar mejoramientos o construcciones de facilidades en la infraestructura de gas del occidente venezolano.

Ahora bien, el inicio de estos trabajos pendientes, se hace sobre la base de lo establecido en la Guía de Gerencia de Proyectos de Inversión de Capital (PDVSA, 1999) sin embargo, es preciso destacar que algunos elementos indicados en este manual como el índice de definición de proyectos (PDRI) y los análisis de riesgos, se aplican poco o casi nunca por parte de las empresas contratistas del sector petrolero del estado Zulia.

La no utilización de estos manuales afecta negativamente el presupuesto original y el tiempo de ejecución en obras de sitios 
remotos, así como cambios de alcance, lo que a su vez ocasiona retraso en la construcción de las instalaciones.

De igual manera, existe desconocimiento del personal en el manejo de la incertidumbre o amenazas lo cual limita la capacidad de respuesta ante el riesgo. Esta desviación genera que la documentación de soporte de decisiones no considere con claridad la evaluación de los riesgos como prioritario en el desarrollo de los trabajos.

Adicionalmente, se observa escasez de personal para emprender este tipo de proyectos, falta de adiestramiento enfocado hacia la planificación y realización de actividades de seguimiento, manejo $y$ control de situaciones que involucren peligros en las zonas donde se desarrollan estos proyectos,

La problemática antes expuesta genera que al llegar el proyecto a la fase de construcción se tenga que realizar modificaciones que no habían sido previstas en la fase de ingeniería, tal vez por los riesgos presentes en el área de ejecución, o por retrasos en la procura de materiales y equipos, por el difícil acceso a la zona de trabajo y/o ausencia de servicios básicos; lo que incrementa los costos debido a la no ejecución oportuna de las actividades propias del proyecto de acuerdo a las características geográficas del área.

De continuar la situación planteada, se pueden cometer errores $u$ omisiones que impliquen retrabajo en los procesos o inclusive paralizaciones de la obra por no haber considerado los riesgos de forma oportuna, por cuanto la falta de gestión de la incertidumbre en los proyectos aumenta la probabilidad de ocurrencia de eventos adversos, que inciden de forma negativa en los costos, cronogramas, integridad del personal participante de la obra, medio ambiente y rendimiento del proyecto.
Adicionalmente, la empresa desaprovecha la oportunidad de fortalecer el desarrollo de sus objetivos estratégicos y crecimiento profesional, comprometiendo así el cumplimento oportuno de las metas trazadas en este tipo de proyectos.

La problemática expuesta plantea la necesidad de dar respuesta a la siguiente interrogante de investigación: ¿Cómo estaría configurado un modelo para la gestión de riesgos en proyectos ejecutados en sitios remotos por las empresas contratistas del sector petrolero del estado Zulia?

En este orden de ideas, el PMBOK (2004) engloba la gestión de riesgos de un proyecto a través de un proceso en el cual puedan relacionarse los distintos actores que hacen vida en el desarrollo del proyecto, teniendo como objetivo aumentar la probabilidad y el impacto de los eventos positivos, y disminuir la probabilidad de los eventos negativos.

Ante esto, se puede definir la gestión de riesgos como el proceso mediante el cual el equipo ejecutor del proyecto define las políticas, estrategias 0 lineamientos necesarios para reducir la incidencia negativa de los factores externos e internos del proyecto y obtener el mayor provecho de los agentes positivos inherentes al mismo. De manera que, para este fin es necesaria la aplicación de herramientas técnicas y estadísticas para garantizar su efectividad y la recuperación post impacto en el caso que ocurra algún evento no deseado.

De igual manera, Lledó y Rivarola (2007) definen a la gestión de riegos como el proceso sistemático de planificar, identificar, analizar, responder y controlar los riesgos del proyecto. Este proceso trata de maximizar la probabilidad de ocurrencia de los eventos positivos y minimizar la probabilidad de ocurrencia de los sucesos adversos al proyecto. La gestión del riesgo está 
conformada por 6 procesos, la planificación de la gestión, identificación de los riesgos, análisis cualitativo de los riesgos, análisis cuantitativo de los riesgos, planificación de las respuestas ante el riesgo y por último el monitoreo y control de los riesgos.

A su vez, Cartay (2010), explica que la gestión de riesgos consiste en anticipar, mediante planes, la evitación del riesgo (de modo proactivo) o seleccionar la respuesta adecuada para atenuarlo cuando ocurre (de modo reactivo).

En lo antes planteado se observa que los autores coinciden en considerar a la gestión de riesgos como un proceso complejo que consiste en identificar, evaluar, analizar, gestionar, responder y controlar los riesgos que puedan presentarse en un proyecto, con el fin de evitar la alteración del uso y manejo de los recursos.

Según Palacios (2005) un proyecto es un conjunto ordenado de actividades, con un principio y un fin, las cuales se encuentran agrupadas y estructuradas dentro de un modelo de planificación, ya que su ejecución requiere de un conjunto de recursos, a fin de cumplir con las limitaciones impuestas en las variables de tiempo, recursos, calidad y costo de manera de lograr objetivos y metas.

Cleland e Ireland (2001) definen a un proyecto como una combinación de recursos organizacionales integrados para crear algo que no existía antes, y eso proporciona una mejor capacidad de desempeño en el diseño y ejecución de las estrategias de la empresa.

Como se puede inferir de las definiciones dadas por los autores antes mencionados, Palacios (2005), Cartay (1996) y Cleland e Ireland (2001), un proyecto es el trabajo realizado por una organización con el objetivo de dirigirse hacia una situación deseada, está comprendido en un tiempo con un inicio y una terminación, bajo un presupuesto determinado, requiere de recursos y es multifuncional.

En resumen, se puede decir que para dar cumplimiento a los objetivos trazados en un proyecto en una zona remota, resulta necesario disponer de ayuda técnica, económica y humana dentro de la empresa, con el fin de poder engranar estos 3 componentes y así, aumentar la probabilidad de éxito y al mismo tiempo minimizar la influencia de agentes negativos en su desarrollo, de allí la importancia de valorar oportunamente los riesgos que pueden atentar contra la vida del proyecto.

En lo que concierne a este artículo la variable en estudio será la gestión de riesgo, la cual se concibe como un proceso presente en todo el ciclo de vida del proyecto, de allí que al determinar la implementación de alguna estrategia para su control sea necesario conocer previamente la situación de los riesgos en las empresas objeto de estudio, este primer paso de diagnóstico de los riesgos se considerará una de las dimensiones de la variable objeto de estudio y su análisis se realizará en función de los siguientes indicadores: valoración del riesgo, control del riesgo y capacidad de respuesta ante el riesgo.

\section{MÉTODO}

El estudio que se llevó a cabo fue de tipo proyectiva, con un diseño de campo, no experimental, transeccional. La población objeto de estudio estuvo constituida por 15 personas, con las siguientes características: de cualquier sexo y edad, profesional de la ingeniería, gerente o responsable de la ejecución de proyectos, con experiencia en el manejo de proyectos ejecutados en sitios remotos. 
Dicha población estaba distribuida por empresas de la siguiente manera: EHCOPEKSA cinco participantes (5); Zulia Industrial Constructions C.A. (ZIC, C.A.) cinco participantes (5); e Industrias Marítima Venezolana (IMV) cinco participantes (5); todas las empresas señaladas se encuentran ubicadas en el estado Zulia.

Los datos se recolectaron mediante la técnica de la encuesta y el instrumento lo constituyó un cuestionario conformado por 47 ítems, con alternativas de respuestas múltiples. La confiabilidad se determinó mediante el coeficiente Alpha Cronbach obteniéndose un valor de 0,72 lo cual indica que es altamente confiable.

Para el análisis de los datos se utilizó la estadística descriptiva, a través de frecuencias absolutas ( $\mathrm{fa}$ ), frecuencia relativa (Fr) y promedio por indicador y la medida de tendencia central la media, la cual se interpretó según baremo expuesto en la tabla 1.

Tabla 1. Baremo de interpretación de los resultados

\begin{tabular}{cccc}
\hline ALTERNATIVA & CONCEPTO & LíMITES & SIGNIFICADO \\
\hline $\mathbf{5}$ & Siempre & $4,21-5,00$ & Muy alta gestión \\
$\mathbf{4}$ & Casi siempre & $3,41-4,20$ & Alta gestión \\
$\mathbf{3}$ & A veces & $2,61-3,40$ & Mediana gestión \\
$\mathbf{2}$ & Casi Nunca & $1,81-2,60$ & Baja gestión \\
$\mathbf{1}$ & Nunca & $1,00-1,80$ & Muy baja gestión \\
\hline
\end{tabular}

\section{RESULTADOS Y DISCUSIÓN}

El análisis de la variable gestión de riesgos en proyectos ejecutados en sitios remotos se realizó considerando las dimensiones siguientes: situación de los riesgos, tipos de riesgos y características de los proyectos.

Con relación a la dimensión situación de los riesgos, cuyos indicadores son: valoración del riesgo, control del riesgo y capacidad de respuesta al riesgo, los resultados fueron los siguientes: Para el indicador valoración del riesgo, se obtuvo un $0 \%$ para las alternativas siempre y nunca, siendo que el mayor porcentaje recayó en las alternativas a veces $(73,33 \%)$; casi nunca (15\%) y casi siempre $(11,67 \%)$.
Asimismo, la media de la valoración del riesgo fue de 3,03, que al ser comparada con el baremo de interpretación la ubica en la categoría de mediana gestión, lo cual permite inferir que la mayoría de los sujetos identifican medianamente las amenazas que afectan el proyecto, igual comportamiento se proyecta en lo referido a la planificación de nuevos riesgos que puedan surgir.

Estos resultados se contraponen a lo expuesto por el autor Lledó y Rivarola (2007) quienes señalan que la valoración del riesgo implica identificar plenamente los riesgos y posteriormente realizar el análisis de los mismos, a fin de establecer los sucesos que puedan afectar seriamente el proyecto, aun cuando su probabilidad de ocurrencia sea muy baja. 
Igual contradicción se presenta al confrontar los resultados de dicha investigación con el PMBOK (2004), donde se describe esta fase como un proceso iterativo, pues se pueden descubrir nuevos riesgos a medida que el proyecto avanza a lo largo de su ciclo de vida. Este proceso se encuentra fundamentado en la incertidumbre, de tal manera que solo los riesgos ya identificados son los que pueden observarse y evaluarse.

De manera similar, el indicador control del riesgo obtuvo 0\% para las alternativas siempre y nunca y sus máximos valores en las alternativas a veces $(48,89 \%$,); casi nunca $(35,56 \%)$ y casi siempre $(15,56)$. La media de 2,80, la ubica en la categoría de mediana gestión, lo cual se traduce en una acción deficiente en cuanto a la aplicación de algunas técnicas estadística para hacer seguimiento a los riesgos identificados en el proyecto y los que pueden presentarse en su desarrollo.

Los resultados planteados reflejan una mediana gestión frente a lo esperado en la administración de un proyecto según lo sustentan los autores PMBOK (2004), Lledó y Rivarola (2007) y Cartay (2010), quienes plantean que el control de riesgo como un proceso permite identificar, analizar $y$ planificar nuevos riesgos, realizar seguimiento a los ya identificados, analizar los ya existentes y realizar seguimiento a las condiciones que activan los planes de contingencia a fin de evitar impactos negativos durante su desarrollo.

En cuanto al indicador capacidad de respuesta ante el riesgo, los resultados arrojaron un $0 \%$ en las respuestas siempre y nunca, con una destacada ubicación para las relativas a las respuestas casi nunca $(42,22 \%) ;$ a veces $(35,56 \%)$ y casi siempre $(22,22 \%)$. La media de 2,80, ubicada en la posición de mediana gestión, refleja que medianamente se desarrollan acciones para mejorar las oportunidades del proyecto ni utilizan herramientas para dar respuestas a los riesgos que impactan a éste.

Esta situación es contrapuesta a lo planteado en el PMBOK (2004), donde se expone que la capacidad de respuesta ante el riesgo aborda a los riesgos según su prioridad, e introduce recursos y actividades en el presupuesto, el cronograma y el plan para la dirección del proyecto, según se requiera.

De igual manera, sucede con lo señalado por Lledó y Rivarola (2007) quienes expresan que es el proceso más importante de la gestión de riesgos, pues es aquí donde se toma la decisión de cómo responder a cada riesgo identificado.

Asimismo, al analizar la dimensión situación de los riesgos su media fue de 2,88 lo cual le ubica, según el baremo de interpretación, en una categoría de mediana gestión, lo cual indica que los gerentes de proyectos desarrollan un proceso de gestión del riesgo, fundamentado en la identificación de los riesgos mediante herramientas de control, sólo medianamente.

Esta situación es contraria a lo expuesto por Lledó y Rivarola (2007) quienes conciben la gestión de riegos como el proceso sistemático de planificar, identificar, analizar, responder y controlar los riesgos del proyecto. El seguimiento de este cuidadoso proceso para maximizar la probabilidad de ocurrencia de los eventos positivos y minimizar la de los sucesos adversos al proyecto al ser medianamente cumplidos solo permitirá alcanzar medianamente los resultados esperados.

Con respecto a la dimensión identificación de los tipos de riesgos, cuyos indicadores fueron financieros, técnicos $y$ ambientales, se presentan a continuación los resultados obtenidos: Con relación a los riesgos financieros se obtuvo una valoración 
de un $0 \%$ para las alternativas siempre y nunca; casi siempre con un $91,11 \%$ y a veces con un $8,89 \%$. Para esta dimensión la media de 3,91 identifica una alta gestión en los aspectos relativos a tasas de interés y sistema cambiario de divisas en los proyectos desarrollados en zonas remotas.

Estos resultados expresan coincidencia con lo expuesto por Córdoba (2006), quien define al riesgo financiero como el impacto sobre el rendimiento financiero de la empresa producto de su apalancamiento financiero, su posición con respecto al tipo de cambio y valores.

De igual manera, Zorrilla (2004) señala que el riesgo financiero es aquel que está vinculado con las posibles pérdidas generadas con las actividades financieras, tales como movimientos desfavorables en los tipos de interés, los tipos de cambios o de precios de las acciones, cambios en las solvencias de los prestatarios o variaciones en los flujos netos de fondos.

Con respecto al indicador riesgos técnicos, los mayores porcentajes se ubicaron en casi siempre con un $66,67 \%$ y a veces con $22,22 \%$, seguido de $11,11 \%$ en casi nunca y $0 \%$ para siempre y nunca. La media de 3,56 indica una alta gestión, es decir, que los gerentes de los proyectos de zonas remotas analizan las especificaciones técnicas y tecnologías y definen los procesos que condicionan el proyecto.

Estos resultados están acordes con lo planteado por Palacios (2005), quien expone que los riesgos técnicos son aquellos relacionados con problemas de diseño, manejo de tecnología, errores durante la ejecución, estimaciones de costos con un grado que no estén bien definidas, programación deficiente $\mathrm{o}$ baja productividad.

El autor también señala que cuando la organización tiene una pobre actitud hacia la calidad suele ser catalogado el riesgo como técnico, debido a que no se tienen bien definidos los métodos, procedimientos o programa de aseguramiento de la calidad que condicionan el desarrollo óptimo del proyecto.

Con relación al indicador riesgos ambientales, el $0 \%$ respondió a las alternativas siempre y nunca, seguido por un $20 \%$ casi nunca; $53,33 \%$ a veces y 26,67 casi siempre. La media fue de 3,07 , que al ser comparada con el baremo de interpretación la ubica en la categoría de mediana gestión. Lo cual indica que medianamente se elaboran planes de contingencia y el análisis estadístico de las condiciones ambientales de las actividades del proyecto, durante su ejecución.

De la situación antes descrita se infiere que en las empresas objeto de estudio se presentan desviaciones en el manejo de los riesgos ambientales, generalmente el tratamiento que se le da a estos riesgos es nulo, sin embargo, en algunas ocasiones se utilizan análisis estadísticos para programar actividades a ejecutar y activar planes de contingencia.

Estos resultados resultan contrarios a los planteamientos de Palacios (2005) y Oñate, Pereira, Suarez y otros (2002), quienes exponen que los riesgos ambientales están asociados con la noción de peligro y son constantes a lo largo de todo el proyecto, por lo que ameritan extrema atención en todas las fases del mismo, exigencia que se acentúa cuando el proyecto es desarrollado en sitios remotos, por las condiciones mismas del contexto, cuyo espacio físico, por lo general, esta caracterizado por irregularidades del terreno y espacios geográficos con inexistencia de servicios públicos, totalmente expuestos a los constantes cambios climáticos que se presenten, lo que redunda en amenazas al éxito del proyecto. 
Asimismo, Palacios (2005), plantea que para estudiar la frecuencia e impacto de los eventos ambientales que caracterizan a la zona de ejecución de la obra son de gran utilidad los análisis estadísticos ya que permiten establecer estrategias o planes de contingencia que minimicen el impacto negativo de los riesgos ambientales.

Por otra parte, al sintetizar el comportamiento de la dimensión tipos de riesgos, cuya media fue de 3,51, que le ubica en la categoría de alta gestión, según el baremo de interpretación. Sin embargo, causa inquietud la valoración de mediana gestión que arrojaron los resultados de la dimensión denominada situación de los riesgos, puesto que indicadores medulares del proceso de gestión de riesgos como lo son valoración del riesgo, control y capacidad de respuesta obtuvieron los resultados más bajos de la investigación.

Obviamente, una inadecuada valoración de los riesgos conlleva a un inadecuado establecimiento de estrategias para su control y por ende la capacidad de respuesta ante el riesgo está limitada, siendo muchas veces inadecuada, lo cual impacta de forma negativa la efectividad de la administración del riesgo por parte de las gerencias de las empresas estudiadas.

Ahora bien, tomando en consideración la postura de García (2010), quien hace énfasis en que la efectividad en la gestión de riesgos depende de la experiencia y capacidad de los participantes encargados de la administración de los mismos en la organización; ante esto, se infiere que exista la posibilidad de que la situación antes planteada sea consecuencia del hecho de que el personal que gestiona el riesgo no posee la experiencia y competencias requeridas, lo cual deja una agenda abiertas a futuras investigaciones referentes a esta temática.
En razón de lo planteado por el autor antes citado, se debe tener una gran habilidad para identificar oportunamente la gama de riesgos y sus posibles respuestas, considerando todos los aspectos del proyecto tales como, ingeniería, finanzas, recursos humanos, aspectos legales y políticos, entre otros.

Con relación a la dimensión características de los proyectos, cuyos indicadores fueron: función del proyecto, presupuesto y localización, se presentan a continuación los resultados obtenidos: El indicador función del proyecto obtuvo respuestas de $0 \%$ para las alternativas algunas veces, casi nunca y nunca, mientras que un $86,67 \%$ y $33,33 \%$ para las alternativas siempre y casi siempre, respectivamente. Este indicador arrojó una media de 4,13 que lo posiciona en una categoría de alta gestión, ante lo cual puede inferirse que en las organizaciones estudiadas se definen claramente los objetivos de los proyectos con respecto a las necesidades de los clientes o función. Este aspecto es de suma importancia ya que puede influir en la rentabilidad del proyecto.

Estos resultados coinciden con lo expuesto por Cartay (2010), quien establece que todo proyecto obedece a un propósito $u$ objetivos definidos que determinan la composición, estructuración y acción del sistema. El autor también plantea que los proyectos se diseñan y operan en función de objetivos bien especificados y que sus salidas o productos finales deben ser congruentes con los objetivos planteados.

De igual forma, Miranda (2005) hace énfasis que todo proyecto debe tener claramente definidos sus objetivos en términos cuantitativos y cualitativos de forma tal que permitan utilizar instrumentos de medición para confrontar las metas 
propuestas y realizar las acciones correctivas cuando sea necesario.

Con referencia al indicador presupuesto, este presentó una valoración de un $0 \%$ en las respuestas nunca, casi nunca y algunas veces; mientras que un $66,67 \%$ y un $33,33 \%$ para las alternativas siempre y casi siempre, respectivamente. La media se ubicó en 4,33 lo cual, según el baremo de interpretación, indica una muy alta gestión en el desarrollo del presupuesto antes del inicio del proyecto, así como la revisión de los históricos de los proyectos ejecutados. Además, puede inferirse que las empresas objeto de este estudio ejercen un adecuado control sobre los factores adversos que genera la complejidad de las zonas donde son ejecutadas las obras.

Estos resultados son coincidentes con la postura de Chamoun (2003), al considerar que el presupuesto es el monto máximo autorizado para el proyecto, contra el cual se mide el desempeño de los costos incurridos en la realización del proyecto, es decir, lo que se tiene disponible para invertir y lo que se puede llevar a cabo con los medios disponibles.

En efecto, una adecuada gestión en el manejo de todos los factores de riesgo que afectan las finanzas del proyecto $y$ comprometen su desarrollo, garantiza la efectividad en la ejecución de la obra así como el retorno de la inversión hecha en el proyecto.

Con respecto al indicador localización del proyecto, este obtuvo un $0 \%$ en la alternativa nunca; $5,33 \%$ en casi nunca y sus máximos valores en las categorías siempre con un $48 \%$ y casi siempre con $33,33 \%$, la media fue de 4,24 , que al ser comparada con el baremo de interpretación le ubica en una posición de muy alta gestión, esta valoración refleja que las empresas objeto de este estudio llevan a cabo una muy alta gestión en cuanto a localización se refiere. Realizan análisis de las diferentes variables que determinan el lugar de desarrollo del proyecto, estudiando los factores externos e internos que pueden afectarlo.

Estos resultados están en línea con lo planteado por Miranda (2005), quien expone que la localización del proyecto deben analizarse las diferentes variables que determinan el lugar donde finalmente se ubicará el proyecto, buscando en todo caso una mayor utilidad o una minimización de costos.

De igual forma, Sapag (2008) precisa que el estudio de localización del proyecto no es más que una evaluación de factores tecnológicos cuyo objetivo general es la elección de la zona que permita mayores ganancias entre las alternativas que se consideran factibles. También enfatiza que dicho estudio no debe centrarse netamente en el aspecto económico; los factores técnicos, legales, ambientales, sociales juegan un papel importante puesto que estos pueden influir de manera positiva o negativa en la rentabilidad del proyecto.

En efecto, al analizar la dimensión características de los proyectos, los resultados arrojaron una media de 4,24 que al ser comparado con el baremo de interpretación indica una muy alta gestión de este proceso. Las empresas objeto de este estudio consideran todos los factores externos e internos que afectan el desarrollo del proyecto y que impactan financieramente su ciclo de vida.

Estos resultados son coincidentes con los planteamientos de Miranda (2005), quien expone que un proyecto presenta características que le permiten su identificación, tales como: los objetivos, las actividades para lograr los objetivos, localización geográfica, ubicación temporal, magnitud de recursos para su ejecución y los 
agentes que se verían afectados por el proyecto. De igual manera, el mismo autor considera que la formulación y desarrollo de un proyecto comprende un conjunto de etapas conocidas como "estudio de preinversión" que incluye una serie de acciones que conducen a la toma de decisiones para la movilización de recursos para el cumplimiento de objetivos determinados.

Finalmente, luego de analizadas las dimensiones que conforman la variable gestión de riesgos, se realizó el análisis de dicha variable con los siguientes resultados: La variable gestión de riesgos presentó una media de 3,54, que al compararse con el baremo de interpretación le ubica en una categoría de alta gestión de los riesgos, sin embargo, muestra debilidades con respecto a la dimensión situación de los riesgos que a pesar de ser clave en el proceso de gestión del riesgo, arrojó la menor valoración $(2,88)$, ante esto se asume que los gerentes de los proyectos desarrollados por las contratistas objeto de estudio planifican el riesgo sin el uso adecuado de técnicas de identificación, ante lo cual pueden surgir imprevistos no considerados. Igual situación se infiere que se presenta con el control de riesgos y la capacidad de respuesta ante el riesgo.

Estos resultados muestran disparidad con los planteamientos del PMBOK (2004) y Cartay (2010), quienes exponen que en la gestión de riesgo el equipo ejecutor del proyecto define las políticas, estrategias o lineamientos necesarios para reducir la incidencia negativa de los factores externos e internos del proyecto y obtener el mayor provecho de los agentes positivos inherentes al proyecto. Ante esto se hace necesaria la aplicación de herramientas técnicas y estadísticas para garantizar la efectividad y la recuperación post impacto, en el caso que ocurra algún evento no deseado.

De igual manera, es de suma importancia velar porque los participantes encargados de la administración de los riesgos posean la experiencia y capacidad que les permitan administrar los riesgos de manera segura, a objeto de garantizar la efectividad y calidad del proyecto, así como disminuir sustancialmente los costos en que se incurren durante su desarrollo.

\section{Modelo para la gestión de riesgo en proyectos ejecutados en sitios remotos por empresas contratistas del sector petrolero del estado Zulia}

El modelo se presenta de acuerdo a lo dispuesto en el PMBOK (2004), referido a las diferentes fases de la gestión de los riesgos, es decir, la planificación de la gestión, identificación de los riesgos, análisis cualitativo y cuantitativo de los riesgos, seguimiento y control del riesgo y planificación de las respuestas ante el riesgo.

Estos procesos interactúan entre si conjuntamente con los procesos de las demás áreas del proyecto, cada fase implica el esfuerzo de una o más personas o grupos de personas, dependiendo de la magnitud del proyecto. Dicho modelo se ilustra en la figura 1. 


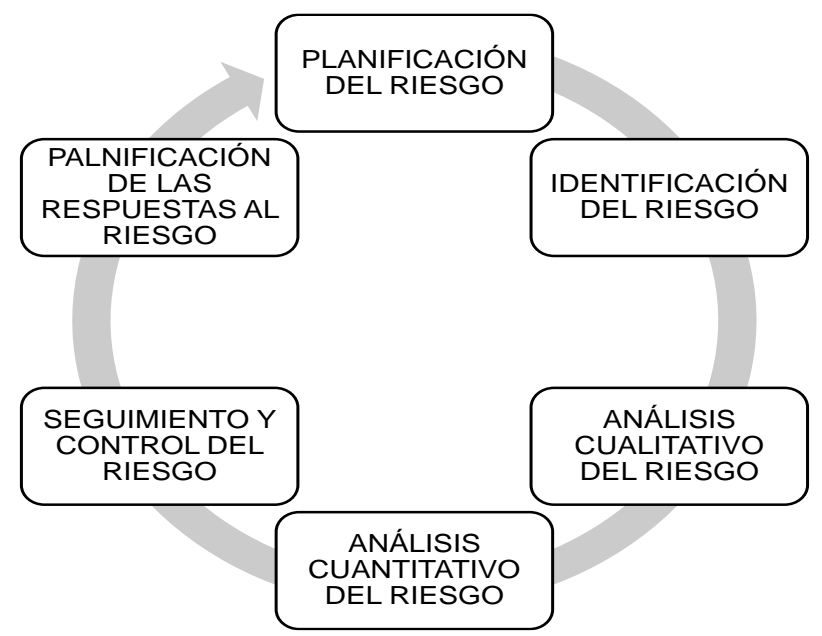

Figura 1. Modelo de Gestión del Riesgo. Fuente: PMBOK (2004)

\section{Fase I. Planificación del riesgo}

En esta fase se decide cómo abordar y llevar a cabo las actividades de gestión de riesgos de un proyecto para garantizar un análisis compatible a la naturaleza del mismo, para tal fin el equipo de proyecto debe contar con una serie de insumos que permitan llevar a cabo una planificación adecuada.

Para proceder a la elaboración del plan de gestión de riesgos el gerente de proyecto realizará reuniones de trabajo para exponer al equipo de proyecto los objetivos del proyecto, donde se expondrán el alcance de los trabajos y se discutirán premisas preliminares de ejecución con base en la documentación técnica disponible y los costos asociados a dicho proyecto.

Posteriormente el gerente de proyecto designará los responsables de la gestión de riesgo según su tipología (financieros, técnicos y ambientales), dicha designación estará basada en el grado de capacitación y conocimiento requerido para tal fin; todo esto se llevará a cabo con apoyo del departamento del recursos humanos a través de un análisis de las habilidades que posea el personal y el potencial de aprender otras, aunado a esto se detectarán necesidades de formación en la materia o en su defecto considerar la incorporación de nuevo personal al equipo ejecutor.

Una vez conformado el equipo de proyecto se procede a la elaboración del plan de gestión de riesgo y se define la metodología y técnicas a utilizar.

El gerente de proyecto conjuntamente con el administrador del contrato estima, sobre la base de los recursos asignados, los fondos necesarios para su inclusión en la línea base de costos y establece los protocolos para la aplicación de la reserva para contingencias y la reserva de gestión.

El gerente de proyecto define cuándo y con qué frecuencia se llevan a cabo los procesos de gestión de riesgos a lo largo del ciclo de vida del proyecto.

De igual manera el planificador conjuntamente con el administrador del contrato establece los protocolos para la utilización de las reservas para contingencias del cronograma y establecen las actividades de gestión de riesgos a incluir en el cronograma del proyecto para la revisión y aprobación del gerente del proyecto.

El equipo de trabajo define como se van a agrupar las causas potenciales de riesgo, y puede utilizar una estructura basada en los 
objetivos del proyecto por categoría o diferentes estructuras RBS si se manejan varios tipos de proyectos. De igual manera, a través de técnicas de análisis de datos (tormenta de ideas, entrevistas, técnica Delphi, entre otras) define la matriz de probabilidad e impacto y calificará la tolerancia al riesgo para vincular la probabilidad de ocurrencia de cada riesgo con su impacto sobre los objetivos del proyecto en caso de que ocurra dicho riesgo. Esto es sometido para la revisión y aprobación del gerente de proyecto.

Una vez compilada la información el gerente conjuntamente con el equipo de proyecto proceden a registrar la data obtenida en los formatos requeridos para tales fines, los cuales pueden variar según la naturaleza del proyecto y es potestad del gerente del proyecto como se auditarán estos procesos. Los formatos propuestos son los siguientes: Plan de Gestión de Riesgos, Identificación y Registro de riesgos, Reporte de Plan de respuesta al riesgo y Reporte de estado de riesgo.

\section{Fase II. Identificación de los riesgos}

En este proceso se determinan los riesgos que pueden afectar de manera positiva o negativa al proyecto. Es el equipo de trabajo liderado por el gerente quien se encarga de la identificación de los riesgos asociados al proyecto. Dicha fase se lleva a cabo durante la ejecución del proyecto.

El objetivo principal de esta fase es documentar las causas, consecuencias y características del riesgo una vez que haya sido identificado. Para tal fin es necesario apoyarse en técnicas de revisión documental y análisis de información; suelen ser de gran ayuda el análisis FODA, tormentas de ideas, entrevistas, técnica Delphi, entre otras.

Una vez recopilada la información a través de las técnicas mencionadas anteriormente el responsable asignado a cada riesgo procede a documentar dicha data en el formato de identificación y registro de riesgos, con el fin de cruzar la información con los registros históricos de la organización a fin de proceder a hacer ajustes acordes al proyecto en desarrollo y optimizar los tiempos de respuesta.

Al contar con toda la información inherente a los riesgos del proyecto, el equipo de trabajo la presenta al gerente de proyecto para realizar una revisión de la misma y proceder a la clasificación y organización de los riesgos, también para depurar la información y excluir aquella que no se considere relevante en cuanto a los eventos que ocurren o puedan ocurrir posteriormente.

\section{Fase III. Análisis cualitativo}

Después de identificados los riesgos se procede a realizar el análisis cualitativo de los mismos ordenándolos en función de su importancia relativa. Para tal fin, es necesario cuantificar los riesgos y esto se consigue mediante un análisis cualitativo.

Además de la cuantificación de los riesgos también es posible (a través de este análisis) determinar la probabilidad de ocurrencia y la magnitud de impacto de éstos sobre el desarrollo del proyecto, para lo cual es necesario que la data generada sea precisa y confiable ya que esto influirá en el grado de entendimiento que pueda tener el equipo de proyecto sobre los riesgos.

Para proceder al desarrollo del análisis cualitativo el equipo de proyecto ejecuta el siguiente procedimiento: El gerente de proyecto selecciona de acuerdo a su criterio y experiencia los registros de riesgos obtenidos en la fase anterior. Posteriormente, toma como base la tabla de probabilidad de ocurrencia de cada riesgo y la tabla de impacto y analiza el efecto 
potencial de los riesgos sobre las metas del proyecto. De igual manera considera el enfoque relativo y numérico, que para esta situación muestra que la contratista procura evitar aquellos riesgos con alto o muy alto impacto.

Seguidamente, para proceder a valorar la probabilidad de ocurrencia del riesgo utiliza la escala relativa y numérica establecida. De igual manera, la terminología impacto se refiere a la magnitud relativa de los efectos que pueden generarse sobre el proyecto una vez detectada la amenaza evaluada.

\section{Fase VI. Análisis cuantitativo}

Esta fase consiste en analizar desde el punto de vista numérico el efecto de los riesgos detectados (ordenados jerárquicamente en el análisis cualitativo) sobre las metas del proyecto. A través de este análisis se puede incorporar información referente a los costos y tiempos de ejecución con el propósito de complementar las acciones a tomar para dar respuesta a cuestionamientos gerenciales desde el punto de vista financiero y técnico.

Para el análisis cuantitativo es necesario contar con el análisis cualitativo ya estructurado y con herramientas de procesamiento de información tales como el análisis de valor esperado, simulación de Monte Carlo y árbol de decisión que permiten evaluar el comportamiento del proyecto en diferentes escenarios obteniendo resultados de mayor precisión. El gerente de proyecto decide si es necesario realizar dicho análisis según el tipo de riesgo y su impacto, puesto que no siempre proporciona respuestas efectivas donde los factores externos presentan fluctuaciones considerables.

\section{Fase V. Seguimiento y control del riesgo}

En el proceso de seguimiento y control se realiza un monitoreo del comportamiento de los riesgos existentes, evaluar la efectividad de las acciones correctivas implementadas y velar por que los riesgos evaluados como poco probables y de bajo impacto se mantengan dentro de la zona de tolerancia establecida por el gerente de proyecto. Para garantizar el éxito de estas acciones se deben llevar registros de los mismos una vez que inicia el proyecto.

El control del riesgo abarca estrategias alternativas tales como: Accionar un plan para contingencias, realizar acciones correctivas y reconfigurar la planificación del proyecto oportunamente.

Para que la fase de seguimiento y control del riesgo sea efectiva el equipo de proyecto debe proceder de la siguiente manera: El miembro del equipo de trabajo responsable de cada riesgo debe informar periódicamente al gerente de proyecto y al resto del equipo de trabajo sobre el comportamiento de la acción implementada, es decir, si se está cumpliendo de forma oportuna y eficaz el plan de respuesta generado o si es necesario reevaluar la situación, es decir, cambiar la estrategia de mitigación del riesgo.

El responsable del riesgo asienta la información obtenida del proceso de monitoreo en el formato de reporte de seguimiento y control de riesgo el cual permite la evaluación actual de los riesgos con sus mecanismos de control o mitigación implantados.

Periódicamente el gerente de proyecto debe realizar reuniones de revisión con el equipo de trabajo donde se discute el estado y evolución de los riesgos existentes, esto con el fin de tomar acciones para enmendar las desviaciones presentes que afecten de manera negativa el desempeño del proyecto. 
De igual forma estas reuniones sirven para alertar sobre posibles amenazas al proyecto para que sean incorporadas al plan de respuesta constituido.

\section{Fase VI. Planificación de las respuestas al riesgo}

En esta fase se toman las decisiones y se ejecutan las acciones para hacer frente a los riesgos identificados en el proyecto. La efectividad de las decisiones tomadas depende de la pertinencia de la información que se cuente, recabada en los análisis previos. Es de vital importancia que el gerente de proyecto cuente con una visión clara y precisa de las amenazas presentes para que la estrategia implementada sea exitosa y sirva como guía para situaciones similares que puedan presentarse posteriormente, esto reduce los tiempos de respuesta para un mejoramiento continuo de los procesos.

Es en esta etapa donde se llevan a cabo una o todas las acciones para cada riesgo principal: realizar algo antes que ocurran los riesgos, propiciar situaciones para generar oportunidades de mejora o disminuir las probabilidades de impacto o amenaza de algún evento.

El proceso de planificación de las respuestas ante el riesgo se refiere al análisis de los diversos riesgos presentes en el proyecto, de igual forma el conjunto de estrategias de respuesta ante las situaciones y por último el plan de respuesta en caso de que la decisión adoptada por el gerente de proyecto sea aceptar el riesgo.

Para la elaboración del plan de respuesta ante el riesgo el equipo de trabajo procede de la siguiente manera: Establece cuáles fueron los riesgos identificados en el proyecto, proceso o área afectada, la probabilidad de ocurrencia y el nivel de impacto del mismo; al igual que la prioridad asignada por el gerente de proyecto.

\section{DISCUSIÓN}

Los autores citados coinciden en que el control de riesgos es un proceso de monitoreo y toma de decisiones constantes, que permiten ejecutar acciones correctivas frente a situaciones que comprometan el desarrollo del proyecto. También resaltan que la comunicación oportuna entre los integrantes del proyecto es importante puesto que permite la reevaluación de lo planeado y crear estrategias en conjunto para hacer frente a los riesgos.

Por consiguiente, las categorías de riesgos deben estar claramente definidas y organizadas según su área y origen para facilitar los planes de respuesta. En los proyectos ejecutados en sitios remotos los riesgos se clasifican de la siguiente manera: riesgo financiero, riesgo técnico y riesgo ambiental.

Con relación al riesgo financiero, los autores citados coinciden que el tener conocimiento de las características mencionadas permite a la organización establecer metas claras y definir las estrategias para poder cumplirlas, de igual forma establece características esenciales y comunes para todo tipo de proyectos.

Con relación a la función del Proyecto, Chamoun (2003) establece que el presupuesto es el monto máximo autorizado para el proyecto contra el cual se medirá el desempeño de los costos incurridos en su realización, es decir, lo que se tiene disponible para invertir y lo que se puede llevar a cabo con los medios disponibles.

Los autores citados convergen que en la localización del proyecto deben considerarse todos los factores externos e internos que puedan afectar el desarrollo del mismo y que impacten financieramente su ciclo de vida. 


\section{CONCLUSIONES}

Con respecto al indicador valoración del riesgo, se pudo constatar que en los proyectos ejecutados en sitios remotos por contratistas petroleras del estado Zulia sólo a veces se identifican las amenazas que afectan el proyecto mediante el uso de técnicas que valoran su impacto. Situación que también se presenta al planificar los nuevos riesgos que pueden surgir.

De igual forma, los resultados del indicador control de riesgos indican poco control mediante técnicas estadísticas para seguimiento de los riesgos identificados y de los que pueden presentarse en el desarrollo del proyecto. Es decir, no se realiza seguimiento a los identificados ni se aborda en su totalidad la identificación, el análisis y la planificación de los nuevos. De igual manera se presentan debilidades al realizar seguimiento a las condiciones que activan los planes de contingencia.

Asimismo, Se infiere que no existe una capacidad de respuesta para abordar los riesgos según su prioridad, al no introducir recursos y actividades en el presupuesto, ni el cronograma ni el plan para la dirección del proyecto, lo que inactiva las posibilidades de mejora del proyecto.

Con respecto a los tipos de riesgos que se presentan en los proyectos desarrollados en las organizaciones abordadas en la investigación, se determinó para los riesgos financieros que casi siempre se evalúan los aspectos relativos a tasas de interés y al sistema cambiario de divisas, lo cual indica que se cumple con un adecuado impacto sobre el rendimiento financiero de la empresa.

Asimismo, los riesgos técnicos poseen una buena ubicación, porque los gerentes de los proyectos siempre analizan las especificaciones técnicas y tecnologías, definiendo los procesos que condicionan el proyecto.

En cuanto a los riesgos ambientales, es preciso detallar que a veces se elaboran planes de contingencia y el análisis estadístico de las condiciones ambientales de las actividades del proyecto; por ello no hay una noción completa de peligro, es decir, identificar la fuente $u$ origen de daños potenciales.

Sin embargo, a pesar de que la dimensión tipos de riesgos obtuvo como resultado una alta gestión, existe la posibilidad de que la gerencia de proyectos pierda la efectividad de la administración del riesgo en razón de la valoración, mediana, obtenida por la dimensión situación de los riesgos a través de sus indicadores.

Asimismo, el indicador función del proyecto permitió inferir que en estas organizaciones siempre se definen claramente los objetivos del proyecto con respecto a las necesidades de los clientes 0 función.

Con relación al indicador presupuesto, este presentó una valoración significativa que indica una muy alta gestión en el desarrollo del presupuesto antes del inicio del proyecto, igualmente la revisión de los históricos de los proyectos ejecutados.

En cuanto al indicador localización del proyecto, sus resultados se traducen en una muy alta gestión desarrollada por las empresas objeto del estudio en relación al proceso de análisis de las diferentes variables que determinan el lugar de ubicación del proyecto.

En razón de los resultados de los indicadores de la dimensión características de los proyectos se infiere una muy alta gestión en este proceso, al cumplir no sólo con la formulación y desarrollo de dichos proyectos, sino también con las etapas conocidas como "estudio de preinversión". 
Finalmente, luego de analizadas las dimensiones de la variable gestión de riesgos, se determinó la estadística de dicha variable, la cual la posicionó en una categoría de alta gestión de los riesgos.

Sin embargo, la variable gestión de riesgos puede verse afectada por las debilidades encontradas en el análisis de la dimensión situación de los riesgos, considerada como vital en el éxito o fracaso de un proyecto.

\section{REFERENCIAS}

Cartay I, (2010). Planificación y control de proyectos. Maracaibo

Cartay, I. (1996). Planificación y Control de Proyectos. Maracaibo - Venezuela

Campero, M. y Alarcón L. (2005) Administración de Proyectos Civiles. Colombia

Chamoun, J. (2003). Administración Profesional de Proyectos - La Guía, Editorial McGraw-Hill Interamericana. Primera Edición México

Coopers y Lybrand. (1997). Los nuevos conceptos del control interno. Informe COSO. Díaz de Santos, Madrid

Córdoba M. (2010). Formulación y evaluación de proyectos ECOE. Ediciones Bogotá

Cleland D. e Ireland L. (2001). Manual Portátil del Administrador de Proyectos. México
D.F. Editorial McGraw-Hill Interamericana. Primera Edición México

García, J. (2010). Ponencia: Gestión de Riesgos en Proyectos de Construcción 1er. Congreso Iberoamericano de Ingeniería de Proyectos

Lledó P. y Rivarola G. (2007). Gestión de proyectos. Prentice Hall - Buenos Aires

Miranda (2005). Gestión de Proyectos: Identificación, Formulación, Evaluación Financiera, Económica, Social, Ambienta, Editorial MM Editores Colombia

Oñate J., Pereira D. y Suarez, F. (2002). Evaluación ambiental estratégica. Ediciones Mundi. Prensa España

Palacios I. (2005). Guía práctica para la identificación, formulación y evaluación de proyectos. Editorial Universidad del Rosario. Colombia

PDVSA (1999) Guías de Gerencia para Proyectos de Inversión de Capital. PDVSA. Caracas. Venezuela

PMBOK) (2004). Guía de los fundamentos de la dirección de proyectos, (2004). Cuarta Edición

Sapag, N. (2008). Proyectos de Inversión. Formulación y evaluación. Primera Edición. Person. Educación. México

Serer, M, (2001) Gestión Integrada de Proyectos

Zorrilla, J. (2004). Administración de riesgos financieros en las PYMES Intangible Capital 